\title{
Detection of Asymmetric PCR Products in Homogeneous Solution by Fluorescence Correlation Spectroscopy
}

BioTechniques 25:706-715 (October 1998)

\author{
Masataka Kinjo \\ Hokkaido University \\ Sapporo, Japan
}

\begin{abstract}
The yield of the double-stranded DNA product (500 bp) of asymmetric PCR with a rhodamine-labeled primer (Rho-primer) was determined in a homogeneous solution using fluorescence correlation spectroscopy (FCS). FCS provides the average number of molecules in a focused volume and the diffusion constant that relates the molecular weight. Since FCS measures the fluctuation of fluorescence intensity in a very small sample volume, the reaction mixture was directly placed on the FCS optical field without any purification procedure after amplification. The result of changing the initial number of templates suggested that elongation of the Rho-primer could be detected by FCS in a PCR mixture containing a single copy of the target gene in the initial condition. Possible scientific applications and perspectives of the proposed approach are discussed.
\end{abstract}

\section{INTRODUCTION}

Since the concept of fluorescence correlation spectroscopy (FCS) was introduced in the early 1970 s $(2,4,10,12)$ and its feasibility was confirmed in the 1990s (20), this novel technique has been applied not only to basic science (22) but also to applied science such as molecular diagnosis $(3,15)$. FCS measurement provides two important analytical parameters in biochemistry: $(i)$ the average number of molecules in the detection field and (ii) the translational diffusion constant of the molecules. The number of molecules and the diffusion constant reflect the chemical reaction and molecular weight and/or structure, respectively. Recent works have demonstrated that FCS has particular advantages in the life sciences because it is sensitive and quantitative $(9,14$, 19,21). Moreover, the composition of fluorescence molecules in a homogeneous solution can be estimated by changing the diffusion constant using FCS, so that this method can be applied to determine the number of DNA fragments in restriction fragment-length polymorphism (RFLP) analysis (8).

Here, we determined the yield of 500 bp DNA in asymmetric polymerase chain reaction (PCR) using FCS as a simple tool for quantification of the diffusion constant. Asymmetric PCR, in which the concentrations of primers are unequal, was introduced to make single-stranded (ss)DNA for direct sequencing or for use as a hybridization probe (5). However, we focused not on the ssDNA product, but on a doublestranded (ds)DNA product in the reac- tion solution. When the concentration of the fluorescence-labeled primer is lower than that of the other primer in amplification, fluorescent dsDNA and nonfluorescent ssDNA can be expected as products of asymmetric PCR. Under ideal conditions, all of the fluorescence-labeled primer (low-concentration primer) is incorporated into dsDNA after the proper number of PCR cycles. From the viewpoint of fluorescence molecules, short ssDNA (fluorescent primer) is elongated into long dsDNA (fluorescent-amplified DNA) during the PCR. The diffusion constant of the elongated DNA is slower than that of the primer, because the diffusion constant simply reflects the molecular weight in the case of a rigid rodlike molecule. Therefore, analysis of the diffusion constant change, which can be obtained by FCS, yields the DNA size change. Thus, the process of the PCR can be determined by diffusion constant measurement by means of FCS. We demonstrate the sensitivity and simplicity of FCS in detecting elongation of dsDNA in fluorescencelabeled primer-based asymmetric PCR (FA-PCR) without any other specific probe. In practice, all of the primer is not exhausted (5) because PCR is not $100 \%$ efficient. Therefore, the reaction mixture contains two fluorescent components after the PCR: the free fluorescent primer and the fluorescent elongated primer (PCR product). The apparent fluorescence signal from the PCR solution contains information about both species that can be directly separated using a two-component model of FCS analysis. The results indicat- 
Table 1. The Yield of Amplified 500 bp DNA with Rho-Primer and the Total Number of Fluorescent Molecules Estimated by FCS Measurements

\begin{tabular}{|c|c|c|c|c|c|}
\hline \multicolumn{2}{|c|}{ Primer Concentration } & \multirow[b]{2}{*}{$\begin{array}{l}\text { Primer } \\
\text { Ratio }\end{array}$} & \multirow[b]{2}{*}{$y^{a}$} & \multirow[b]{2}{*}{$N^{b}$} & \multirow[b]{2}{*}{ Totalc } \\
\hline $\begin{array}{l}\text { Primer } 1(\mathrm{nM}) \\
\text { (Rho-primer 1)d }^{\mathrm{d}}\end{array}$ & $\begin{array}{l}\text { Primer } 2 \text { (nM) } \\
\text { (Rho-primer 2)d }\end{array}$ & & & & \\
\hline 800 & $400^{d}$ & $1: 0.5$ & 0.30 & 900 & 120 \\
\hline 800 & $40^{d}$ & $1: 0.05$ & 0.60 & 90 & 24 \\
\hline 800 & $4^{d}$ & 1:0.005 & 0.52 & 8 & 2.1 \\
\hline $400^{d}$ & 800 & $0.5: 1$ & 0.35 & 863 & 140 \\
\hline $40^{d}$ & 80 & $0.05: 1$ & 0.21 & 76 & 8.4 \\
\hline $4 d$ & 800 & $0.005: 1$ & NDe & 9 & NDe \\
\hline \multicolumn{6}{|c|}{$\begin{array}{l}\text { In the fitting, } \tau_{\text {free }}(0.17 \mathrm{~ms}) \text { and } \tau_{\text {poly }}(1.98 \mathrm{~ms}) \text { were fixed to reduce free } \\
\text { parameters. }\end{array}$} \\
\hline \multicolumn{6}{|c|}{ aThe yield of elongated rhodamine-labeled primer. } \\
\hline \multirow{2}{*}{\multicolumn{6}{|c|}{$\begin{array}{l}\text { bThe average number of fluorescence molecules in the focused field. } \\
\text { cTotal yield was calculated with the initial concentration of fluorescent primer } \\
\text { and the yield of elongated rhodamine-labeled primer }(y) \text {. }\end{array}$}} \\
\hline & & & & & \\
\hline \multicolumn{6}{|c|}{ dConcentration of rhodamine-labeled primer. } \\
\hline
\end{tabular}

ed that the combination of FCS and FAPCR could detect the target gene at the single-copy level at the initial concentration in $25 \mu \mathrm{L}$ of solution without using any separation or purification method such as gel electrophoresis, column chromatography or ligandbinding selection.

\section{MATERIALS AND METHODS}

\section{Asymmetric PCR}

A set of primers (primer 1: $5^{\prime}$-GAT GAG TTC GTG TCC GTA CAA CTG G-3', primer 2: 5'-GGT TAT CGA AAT CAG CCA CAG CGC C-3') was purchased from Hokkaido System Science (Sapporo, Japan). Rhodamine-labeled primers (Rho-primer 1 and Rho-primer 2) were from Takara Shuzo (Tokyo, Japan). Typical PCR was carried out in a $25-\mu \mathrm{L}$ vol with $2.5 \mathrm{U}$ of AmpliTaq Gold $^{\mathrm{TM}}$ DNA Polymerase (PE Applied Biosystems, Foster City, CA, USA), 1× associated buffer [10 mM Tris- $\mathrm{HCl}, \mathrm{pH}$ $8.3,50 \mathrm{mM} \mathrm{KCl}, 1.5 \mathrm{mM} \mathrm{MgCl}_{2}$ and $0.001 \%(\mathrm{wt} / \mathrm{vol})$ gelatin], $0.2 \mathrm{mM}$ $\mathrm{dNTP}, 0.8 \mu \mathrm{M}$ primer $1,0.04 \mu \mathrm{M}$ Rhoprimer 2 and whole $\lambda$ phage DNA (48.5 $\mathrm{kbp}, 3.2 \times 10^{7} \mathrm{Da}$; as $\left.1 \mathrm{bp}=660 \mathrm{Da}\right)$ as a template. The concentration of the template was varied from $1 \mathrm{ng} / 25$ $\mu \mathrm{L}-0.1 \mathrm{fg} / 25 \mu \mathrm{L}$, which corresponds to from $1.9 \times 10^{7}-1.9$ molecules $/ 25 \mu \mathrm{L}$. A sequence of $500 \mathrm{bp}$ was amplified with a Model PC-700 Programmable Thermal Controller (Astec, Fukuoka, Japan). After preincubation at $96^{\circ} \mathrm{C}$ for $15 \mathrm{~min}$, each PCR cycle was carried out with annealing at $55^{\circ} \mathrm{C}$ for $1 \mathrm{~min}$, extension at $72^{\circ} \mathrm{C}$ for $2 \mathrm{~min}$ and denaturation at $96^{\circ} \mathrm{C}$ for $1 \mathrm{~min}$. An adequate amplified reaction sample $(10 \mu \mathrm{L})$, obtained after 50 cycles, was placed on a Lab-Tek ${ }^{\circledR}, 8$ -

$$
G(t)=1+\frac{1}{N}\left[\left\{\frac{1-y}{1+\frac{t}{\tau_{\text {free }}}} \sqrt{\frac{1}{1+s^{2} \cdot \frac{t}{\tau_{\text {free }}}}}\right\}+\left\{\frac{y}{1+\frac{t}{\tau_{\text {poly }}}} \sqrt{\frac{1}{1+s^{2} \cdot \frac{t}{\tau_{\text {poly }}}}}\right\}\right]
$$

Equation 1.

Chambered Cover Glass (Nalge Nunc International, Naperville, IL, USA) for FCS measurement and then subjected to agarose gel electrophoresis.

\section{FCS Measurement}

Although details of the FCS experimental setup have been described before $(7,8,16-18)$, Figure 1 presents the schematic detection field to help understand the concept of FCS. FCS measurement was performed using a ConfoCor ${ }^{\circledR}$ Fluorescence Correlation Measurement System (Carl Zeiss Jena $\mathrm{GmbH}$, Jena, Germany) with an objective (C-Apocromat 40× 1.2W; Carl Zeiss) at room temperature. The sample droplet on the cover glass was set on the objective with a small cover to prevent solvent evaporation during measurement (1 min).

Although the intensity distribution of a focused laser beam is Gaussian, fluorescence observation volume is represented by a quasi-cylindrical profile (16). Hence, we can suppose that the laser beam focused on sample solution produced a very tiny cylindrical field in which the fluorescence particles moved in and out due to Brownian motion (Figure 1, upper right). Then the fluctuation of fluorescence intensity caused by the fluctuation in the number of fluorescent particles in the tiny field could be monitored. The fluctuation of intensity was analyzed using an autocorrelation function and provided the average number of molecules and the translational diffusion time of the molecules in the focused field. The fluorescence autocorrelation function $(G(t))$ was fitted to a simple two-component model (18) with the average number of fluorescence molecules $(N)$, the translational diffusion time of the free primer $\left(\tau_{\text {free }}\right)$ and that of the extended primer $\left(\tau_{\text {poly }}\right)$, according to Equation 1 with $\tau_{\text {free,poly }}=$ $w_{\mathrm{O}}{ }^{2} / 4 \mathrm{D}_{\text {free, poly }}$ and $\mathrm{S}=w_{\mathrm{o}} / z_{\mathrm{o}}$, where $y$ is the fraction of the extended primer, $w_{\mathrm{O}}$ is the radius of the detection field (volume element), $2 z_{\mathrm{O}}$ is the field length and $\mathrm{D}_{\text {free }}$ and $\mathrm{D}_{\text {poly }}$ are the translational diffusion constants of free and extended primers, respectively. The diffusion time $(\tau)$ obtained through the volume element is related to the diffusion constant, so that we use the term diffusion 
time in the Results and Discussion.

The data analysis was performed using the nonlinear least-squares method with the FCS ACCESS computer program (EVOTEC BioSystems $\mathrm{GmbH}$, Hamburg, Germany).

\section{RESULTS AND DISCUSSION}

The volume of the detection field was defined by measurement of the diffusion time of rhodamine $6 \mathrm{G}$ (data not shown) as an authentic sample. The ratio of the radius to the length of the detection area, $\mathrm{S}$, was about 5.2, and the volume element was calculated as $1 \times 10^{-15} \mathrm{~L}$.

To determine the proper ratio of the primer set in asymmetric PCR, a preliminary experiment was performed. Six primer ratios were tested, 3 for primer 1:Rho-primer 2 (800:400 nM, 800:40 $\mathrm{nM}$ and 800:4 $\mathrm{nM}$ ) and 3 for Rho-primer 1:primer 2 (400:800 nM, 40:800 $\mathrm{nM}$ and 4:800 $\mathrm{nM}$ ) in the pres- ence of $1 \mathrm{ng}$ of template. After $50 \mathrm{cy}-$ cles of PCR, $10 \mu \mathrm{L}$ of the reaction solution were set on the cover glass and then measured by FCS for $1 \mathrm{~min}$. Figure 1 shows typical fluorescence auto- correlation functions. The translational diffusion time of Rho-primer $2\left(\tau_{\text {free }}=\right.$ $0.164 \mathrm{~ms})$ was defined from a control sample that had not been subjected to a thermal cycle (Figure 1, O). Another

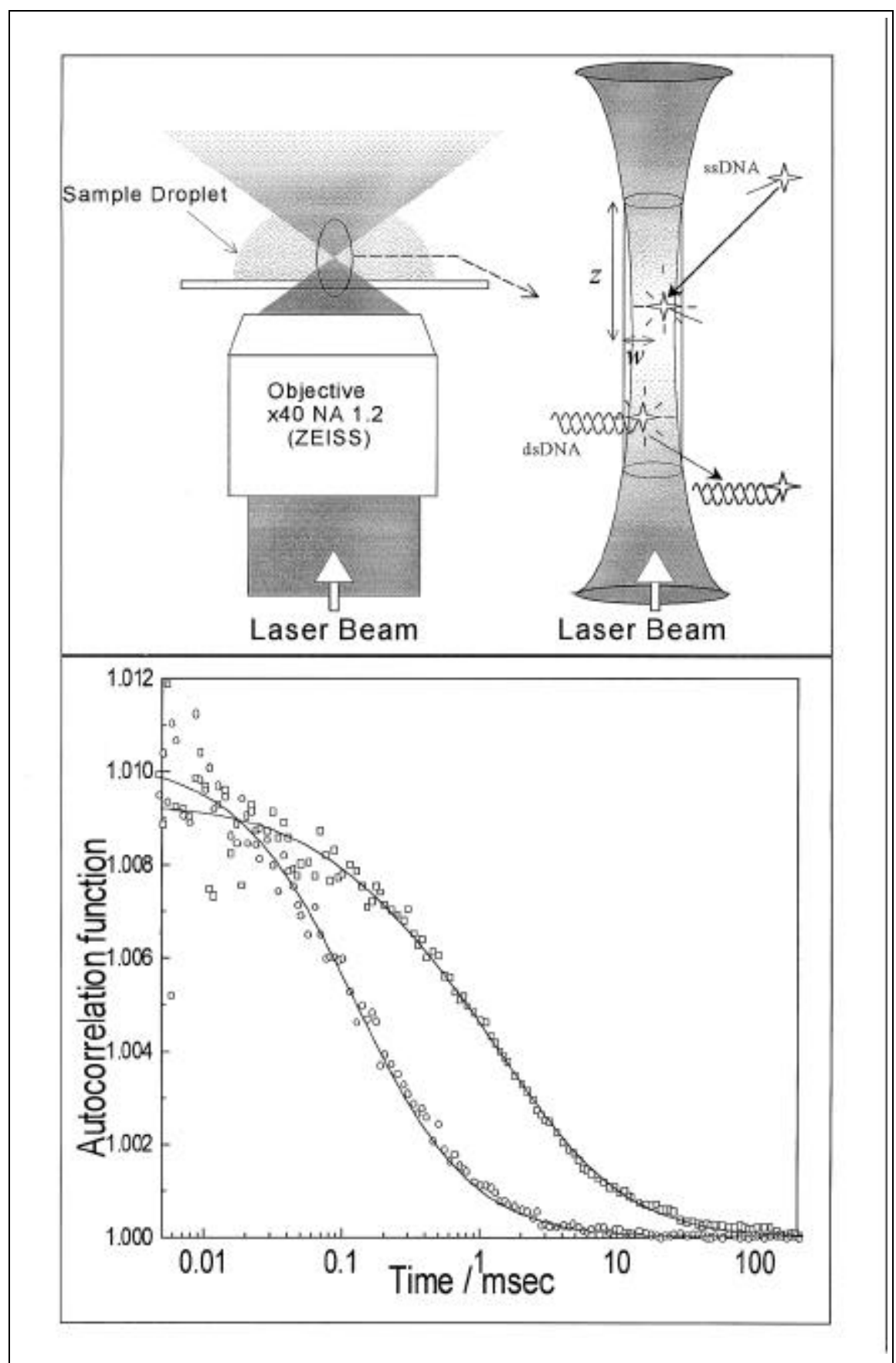

Figure 1. (Upper panel) Schematic diagram of FCS detection apparatus. Left: a laser beam is focused on the sample droplet on the cover glass. Right: the volume element is indicated by the cylindrical region in the focused volume with radius $(w)$ and length $(2 z)$. Stars represent fluorescent probes. (Lower panel) Autocorrelation function of free Rho-primer $(\mathrm{O})$ and asymmetric PCR product $(\square)$ in the condition of primer 1:Rho-primer $2(800: 40 \mathrm{nM})$ after 50 cycles of PCR. The curves were obtained by fitting with Equation 1 and give the translational diffusion time of the free Rho-primer $\tau_{\text {free }}=0.164 \mathrm{~ms}$, and the translational diffusion time and the yield of amplified DNA $\tau_{p o l y}=1.98$ and $y=0.6$, respectively. 
control sample, minus enzyme but with the thermal cycle, was also measured (data not shown), and the result was $0.169 \mathrm{~ms}$. The translational diffusion time of 500-bp DNA (PCR products) was evaluated from curve fitting of autocorrelation data, giving $\tau_{\text {poly }}=1.98$ ms (Figure $1 \square$ ). The value agreed with the 500-bp length DNA according to theoretical calculation of the translational diffusion constant of rodlike molecules (13). Therefore, the amplified product was well identified from the change in the autocorrelation function. Due to the different translational diffusion times of the free primer and the amplified primer, we were able to quan- tify the fractions of amplified products.

In the analysis, the values of $\tau_{\text {poly, }}$ $\tau_{\text {free }}$ and $S$ were fixed in the fitting to reduce the number of free parameters and clearly separate the ratio of free-to-extended primers $(y)$. Although the free primer and PCR products were not physically separated after the thermal cycle, it was possible to measure the fractions of products by this analysis (Table 1). In the case of primer 1:Rhoprimer $2(800: 40 \mathrm{nM})$, the ratio between the remaining free primer and PCR products $(y=0.6)$ was better than with the others. At the primer ratio of $800: 400 \mathrm{nM}$, the total product of $500 \mathrm{bp}$ was better than at other primer ratios;

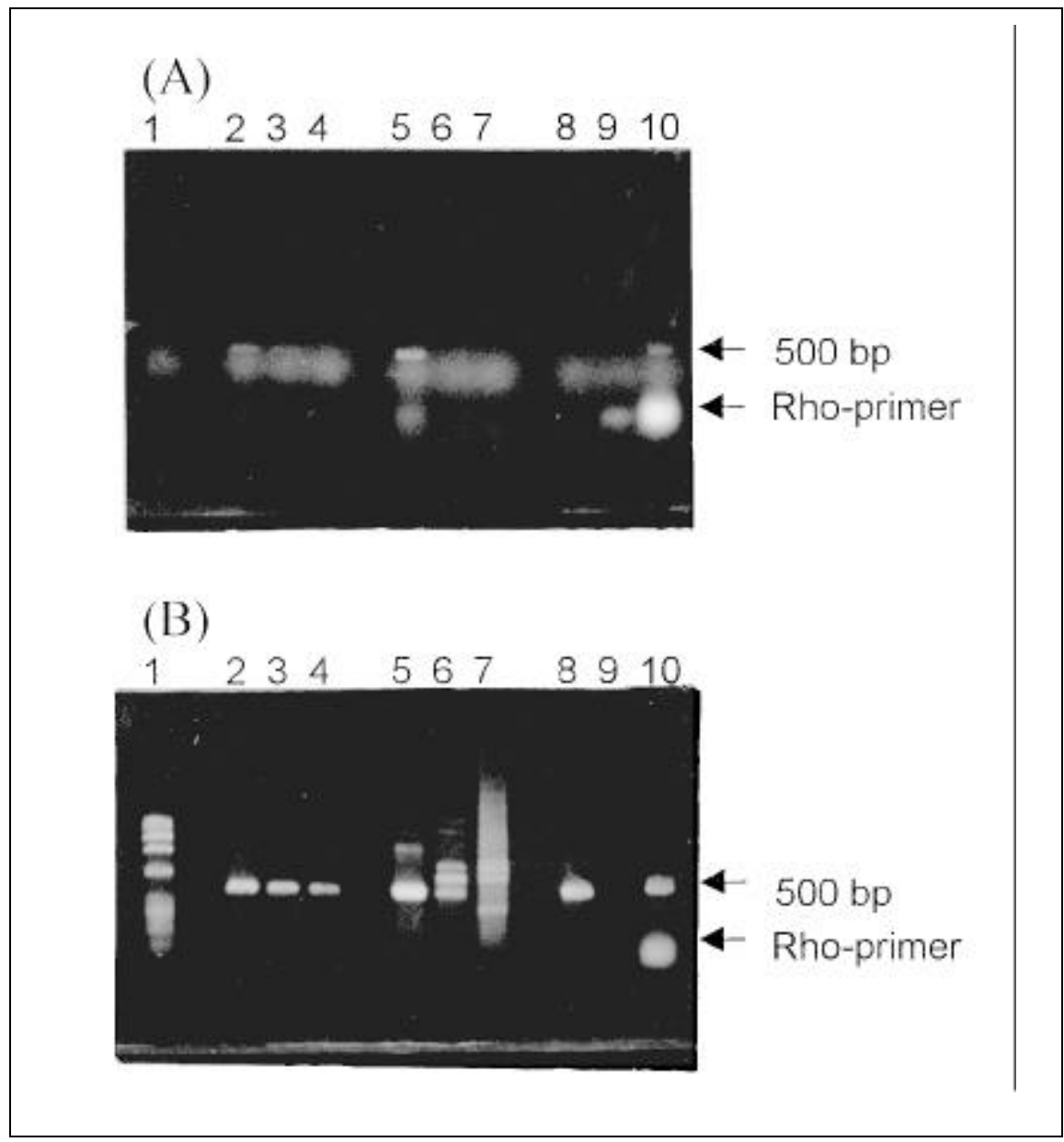

Figure 2. Characterization of asymmetric PCR products with different primer ratios by agarose gel electrophoresis. Asymmetric PCR solution after 50 cycles $(3 \mu \mathrm{L})$ was applied to $1.4 \%$ agarose gel. (A) Without ethidium bromide staining. (B) After ethidium bromide staining. Lane 1, marker ( $\phi$ X174 digested with HaeIII). Lane 2, primer 1:Rho-primer 2 (800:400 nM). Lane 3, primer 1:Rho-primer 2 (800:40 nM). Lane 4, primer 1:Rho-primer 2 (800:4 nM). Lane 5, Rho-primer 1:primer 2 (400:800 nM). Lane 6, Rho-primer 1:primer $2(40: 800 \mathrm{nM})$. Lane 7, Rho-primer 1:primer $2(4: 800 \mathrm{nM})$. Lane 8, Control, PCR with normal primers. Lane 9, Control, Rho-primer 2. Lane 10, Control, PCR with Rho-primer 1 and Rho-primer 2 . The positions of $500 \mathrm{bp}$ and Rho-primer 2 are indicated by arrows. The faint, broad band between $500 \mathrm{bp}$ and the Rho-primer is bromophenol blue dye. 
however, approximately $70 \%$ of the free primer still remained. Although it was expected that all of Rho-primer 2 would be incorporated into the PCR products at the primer ratio of $800: 4 \mathrm{nM}$, only about half of the labeled primer 2 ( $y=$ 0.52 ) could be incorporated, so the yield of product was not better than at the ratio of 800:40 nM. Conversely, in the case of Rho-primer 1:primer 2 (400:800 nM, 40:800 nM and 4:800 $\mathrm{nM})$, yields of PCR products were less than 0.35. Detailed examination of the kinetics of the hybridization process would help to clarify the reason for this.

The amplified target products (500 bp) were also analyzed by agarose gel electrophoresis after being quantified by FCS measurement. Figure 2 shows the results. A fluorescent band of rhodamine at $500 \mathrm{bp}$ was detected without ethidium bromide staining in all cases (Figure 2A). The PCR products were also confirmed by ethidium bromide staining after electrophoresis (Figure 2B). ssDNA of 500 bases was not clear in the gel because the fluorescence in- tensity of ssDNA was less than that of dsDNA by this staining method. In the case of Rho-primer 1:primer 2 (40:800 and 4:800 $\mathrm{nM}$ ), smears and nonspecific bands were detected in the gel (Figure 2, lanes 5-7). Conversely, in the case of primer 1:Rho-primer 2 (800:400 nM, $800: 40 \mathrm{nM}$ and 800:4 nM), the 500-bp band was clearly detected, and its intensity was reduced according to the ratio from 1:0.5-1:0.005 (Figure 2, lanes $2-4)$. These results showed good agreement with FCS determination (Table 1). The ratio of primer 1:Rho-primer 2 (800:40 nM) was used in further experiments in this work because the yield of 500 bp $(y=0.6)$ was better than with other ratios. FCS-based, FA-PCR detection would have the advantages of quantitative accuracy and simplicity. One can detect a target gene by 1-min FCS measurement with only $10 \mu \mathrm{L}$ of reaction solution after FA-PCR amplification. It would be interesting if a low copy number of template genes could be amplified by FA-PCR and then detected by FCS. To investigate the sensitivity of the method, the concentration of the template was changed from $1.9 \times 10^{7}-1.9$ molecules in $25 \mu \mathrm{L}$ of reaction mixture.

The autocorrelation function $G(t)$ of Rho-primer 2 shifted toward a longer correlation time with the increase in the template number after 50 thermal cycles (Figure 3 ). This corresponded to the increase of extended Rho-primer 2. The yield and the number of FA-PCR products were calculated according to Equation 1. The average number of fluorescence molecules in the optical field is plotted in the inset of Figure 4. No specific influence on the number of labeled DNA molecules was observed, so that specific or nonspecific adhesion or partitioning to the surface of the tube and/or glass was not detected in this experiment. The yield of the product was dependent on the initial number of template molecules from $10^{2}$ to $10^{4}$ molecules/25 $\mu \mathrm{L}$ (Figure 4 ), which can be used as the quantification range. Even in the initial presence of 190 template molecules in $25 \mu \mathrm{L}$, the correlation decay of amplified DNA was separated,

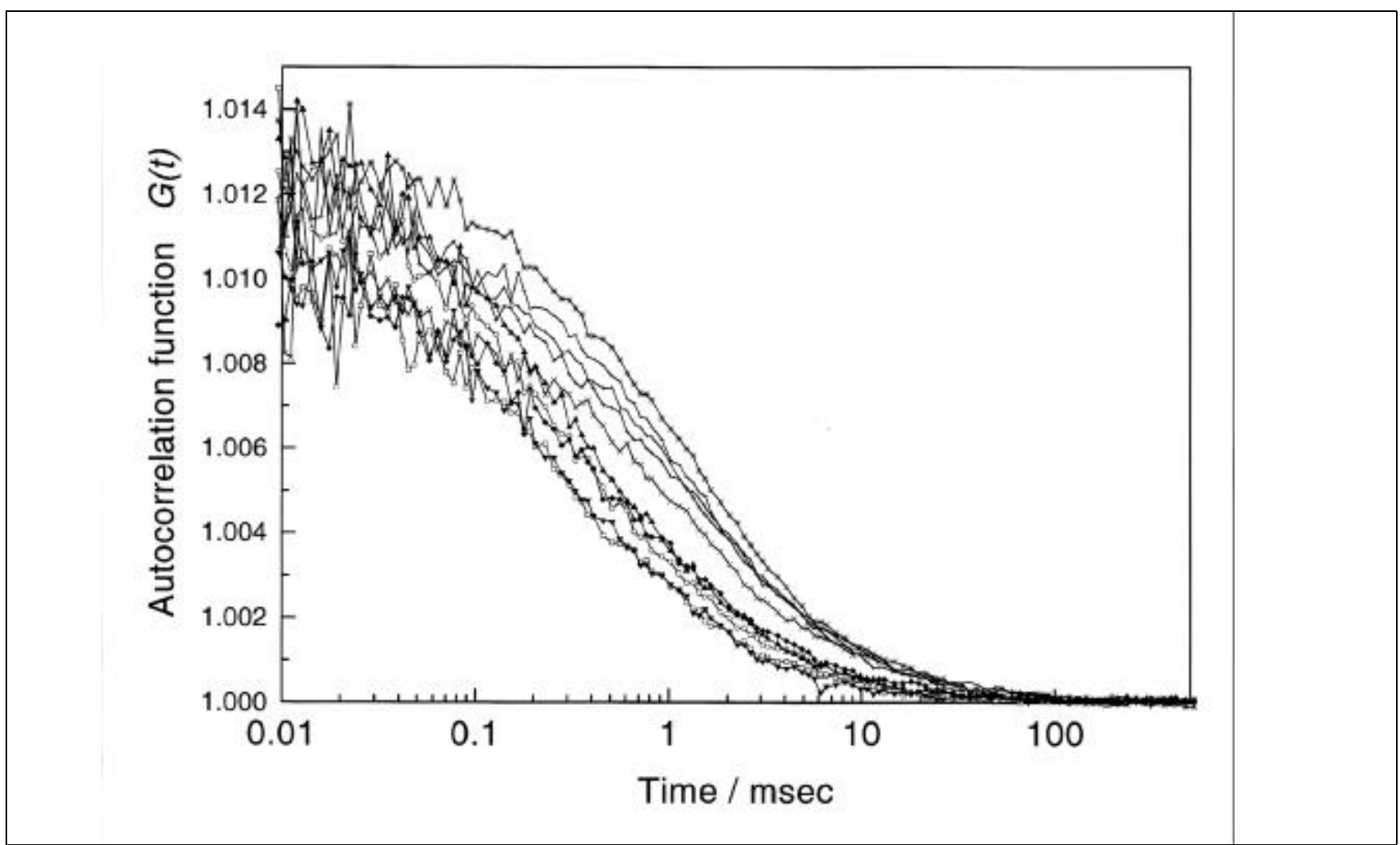

Figure 3. Autocorrelation function of fluorescence-labeled primer in asymmetric PCR for different template numbers. Autocorrelation function was measured with $10 \mu \mathrm{L}$ of FA-PCR solution after 50 cycles. $\square$, O: Blank; $\mathbf{\Delta}: 1.9 ; \boldsymbol{\nabla}: 1.9 \times 10 ; \bullet: 1.9 \times 10^{2} ;+: 1.9 \times 10^{3} ; \mathrm{X}: 1.9 \times 10^{4}, *: 1.9 \times 10^{5} ;-: 1.9 \times 10^{6}$, : $1.9 \times 10^{7}$ template molecules in $25 \mu \mathrm{L}$. 
and it could be detected clearly. A plateau effect was observed with over $10^{5}$ template molecules after 50 cycles. Although a number of factors could have contributed to the plateau effect, it was simply related to the number of cycles. Therefore, the quantification range will be optimized by reducing the number of thermal cycles for a higher concentration of initial template molecules.

Detection of target genes using FCS has been reported recently $(7,14,19$, 21). Nucleic acid sequence-based amplification (NASBA) was combined with FCS and has proven to be a useful detection method for human immunodeficiency virus (HIV) diagnosis in plasma (14). It is an altered PCR method; however, it uses two primers and an extra fluorescence-labeled primer as a probe in the presence of three enzymes, such as T7 RNA polymerase, reverse transcriptase and RNase H. A simpler method has been reported by the same group (21), amplified probe extension (APEX) detection by FCS, but it still uses an extra fluorescence-labeled primer as a probe se- quence besides a forward primer and a reverse primer set. A time shift of the autocorrelation function was observed after 26 cycles in that experiment. To enhance the sensitivity of the FCS method in the PCR technique, we have developed the fluorescence random-labeling method using a fluorescent mononucleotide in PCR and successfully quantified the target gene after 20 cycles of amplification (7). Although the random-labeling method was carried out with a rather small number of amplification cycles, the physical separation process of unincorporated fluorescence monomers from PCR products with a spin column was still used. Conversely, the FCS-based, FA-PCR detection method described here requires only a fluorescence-labeled primer in reaction condition without a physical separation method such as gel filtration.

Fluorescence quenching-based PCR quantification, such as fluorescence energy-transfer analysis, was reported previously $(6,11)$. Although this technique is now commercially available, the reaction contains two kinds of fluo- rescence dyes in one probe sequence besides a PCR primer pair (such as the NASBA and the APEX methods described above). The design of the probe oligonucleotide should consider not only a sequence that does not compete with primers, but also the melting temperature $\left(\mathrm{T}_{\mathrm{m}}\right)$. Moreover, the position between donor and acceptor dyes affects the efficiency of increase of fluorescence intensity and the $5^{\prime}$ nuclease activity of the polymerase. Another fluctuation spectroscopy method (fluorescence cross-correlation spectroscopy) was reported for detection of a specific target gene in a homogenous solution (1). Although the cross-correlation method detected the target sequence at very low concentrations, such as $10^{-12} \mathrm{M}$ without amplification, this method still requires two kinds of fluorescence probes in experimental solution. From the viewpoint of simplicity, the FCS-based PCR quantification method offers a major advantage over the energy-transfer and cross-correlation methods. The fidelity and sensitivity of PCR are strongly dependent

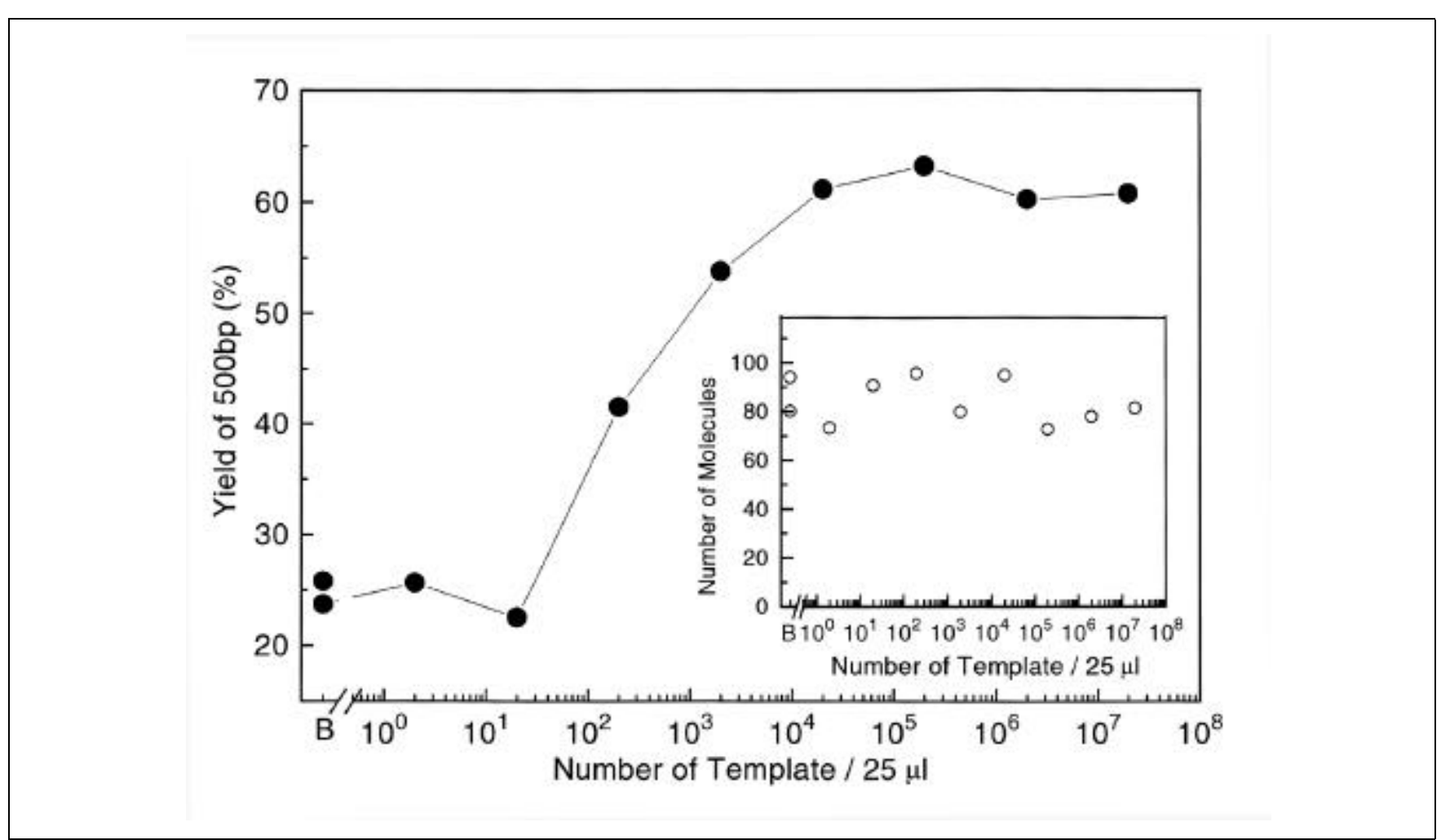

Figure 4. The yield of the asymmetric PCR product $(500 \mathrm{bp})$ was determined in the presence of different numbers of templates after 50 cycles. The yield $(y)$ and the number of molecules $(N)$ were calculated according to the two-component model. The yield is indicated against the input Rho-primer, and the average number of fluorescence molecules in the focused field is plotted (insert). B: control experiment without template. 
on the type of enzyme (Pol I type or $\alpha$ type), selection of primer sequence and reaction conditions. Once these conditions can be fixed, FCS-based, FA-PCR can be immediately applied to the ordinary PCR assay with slight modification of the primer ratio. Therefore, routine PCR conditions will be easily modified to conditions for FA-PCR. Moreover, other amplification methods, such as reverse transcription (RT)-PCR can be modified for this method, and it should be possible to quantify the mRNA in single cells in the future.

In gel electrophoresis and column chromatography, the PCR product is separated from the primer and by-products. However, the target product is diluted during the electrophoresis and extraction procedures from the gel materials and then has a chance to come into contact with nuclease, leading to degradation. Conversely, the FCS method is convenient for sample collecting after measurement; a sample droplet is placed on a cover glass, and FCS is a noninvasive method, so the PCR solution is not diluted or destroyed during measurement. Although the resolution of gel electrophoresis is higher and can detect a single nucleotide difference in length, the very rapid analysis time (1 min) of FCS measurement compared with gel electrophoresis $(1 \mathrm{~h})$ will be advantageous for massive specimen analysis (for example, in diagnosis and screening). The screened sample from FCS can be immediately subjected to detailed analysis, such as gel electrophoresis, to confirm the FCS results or to analyze the nucleotide sequence. Moreover, the collected sample can be used in further experiments, (for example, as a hybridization probe). If one need not collect the amplified sample after FCS measurement, it can be discarded without a risk of carryover contamination because FCS is a noncontact measurement method.

As FCS is noninvasive and allows direct measurement, the PCR volume is dependent on the measurement volume needed for FCS. Our current measurements of FCS were carried out with a $10-\mu \mathrm{L}$ sample volume, though the sample volume can theoretically be reduced to a volume element of the order of $10^{-15}$ L. However, the PCR volume can be reduced to $1 \mu \mathrm{L}$, and thus, it would have a great advantage in comparison with other methods from an economical point of view. Moreover, FCS is based on fluorescence microscopy, and the detection field is set on the objective, so that this method will be able to quantify specific RNA and DNA from in situ PCR.

We used a two-component model for analysis of the PCR solution and obtained good results. However, many DNA species might be present in other experiments. In such cases, species of DNA can be well separated by conventional gel electrophoresis. Although the gap in the correlation curve from the model function to observed data of FCS could suggest some other components distributed in the solution, the simple two-component model seems to be the limit in such cases. Consequently, when more detailed quantitative analysis is necessary, multicomponent FCS analysis (13) should be developed to obtain the distribution function using a computer program such as CONTIN (Max Planck Institute for Biophysical Chemistry, Göttingen, Germany).

Without using a physical separation procedure, this method is noninvasive, noncontact and uses a small sample volume. Physical handling of such a small sample volume can be done by using a capillary tube or by using a small sample pit on a glass surface covered by a thin cover glass or by film for on-line analysis. With these properties and improvements, it is straightforward to use, and the process used to monitor amplification is simple (on-line monitoring) and can be used with an automatic detection system (robotics). We therefore conclude that FCS-based, FAPCR quantification has advantages in terms of sensitivity, quantitative precision and simplicity. We are hopeful that the method will be useful not only for rapid screening, but also to open a new field of molecular diagnosis.

\section{ACKNOWLEDGMENTS}

The author thanks Dr. G. Nishimura (RIES, Hokkaido University) for critical discussion about FCS measurement and analytical methods.

\section{REFERENCES}

1.Castro, A. and J.G.K. Williams. 1997. Single-molecule detection of specific nucleic acid sequence in unamplified genomic DNA. Anal. Chem. 69:3915-3920.

2.Ehrenberg, M. and R. Rigler. 1974. Rotational brownian motion and fluorescence intensity fluctuations. Chemical Physics 4:390401.

3.Eigen, M. and R. Rigler. 1994. Sorting single molecules: application to diagnostics and evolutionary biotechnology. [Review]. Proc. Natl. Acad. Sci. USA 91:5740-5747.

4.Elson, E.L. and D. Madge. 1974. Fluorescence correlation spectroscopy. I. Conceptual basis and theory. Biopolymers 13:1-27.

5.Gyllensten, U.B. and H.A. Erlich. 1988. Generation of single-stranded DNA by the polymerase chain reaction and its application to direct sequencing of the HLA-DQA locus. Proc. Natl. Acad. Sci. USA 85:7652-7656.

6.Heid, C.A., J. Stevens, K.J. Livak and P.M. Williams. 1996. Realtime quantitative PCR. Genome Res. 6:986-994.

7.Kinjo, M. 1998. Quantitative analysis by the polymerase chain reaction using fluorescence correlation spectroscopy. Anal. Chim. Acta. 365:43-48.

8.Kinjo, M. and G. Nishimura. 1997. Fluorescence correlation spectroscopy as a detection tool of point mutation in genes. Bioimaging 5:134-138.

9.Kinjo, M. and R. Rigler. 1995. Ultrasensitive hybridization analysis using fluorescence correlation spectroscopy. Nucleic. Acids. Res. 23:1795-1799.

10.Koppel, D.E. 1974. Statistical accuracy in fluorescence correlation spectroscopy. Physical Review A 10:1938-1945.

11.Livak, K.J., S.J.A. Flood, J. Marmaro, W. Giusti and K. Deetz. 1995. Oligonucleotides with fluorescent dyes at opposite ends provide a quenched probe system useful for detection PCR product and nucleic acid hybridization. PCR Methods Appl. 4:357-362.

12.Magde, D., E.L. Elson and W.W. Webb. 1974. Fluorescence correlation spectroscopy. II. An experimental realization. Biopolymers 13:29-61.

13.Nishimura, G., R. Rigler and M. Kinjo. 1997. Number analysis of fluorescence correlation spectroscopy for the cleaving process of fluorescence labeled DNA. Bioimaging 5:129-133.

14.Oehkenschläger, F., P. Schwille and M. Eigen. 1996. Detection of HIV-1 RNA by nucleic acid sequence-based amplification combined with fluorescence correlation spectroscopy. Proc. Natl. Acad. Sci. USA 93: 12811-12816.

15.Rigler, R. 1995. Fluorescence correlations, single molecule detection and large number screening-applications in biotechnology. J. Biotechnol. 41:177-186.

16.Rigler, R., Ü. Mets, J.Widengren and P. Kask. 1993. Fluorescence correlation spectroscopy with high count rate and low background: analysis of translational diffusion. Eur. Biophys. J. 22:169-175.

17.Rigler, R. and J. Widengren. 1990. Ultrasensitive detection of single molecules by fluorescence correlation spectroscopy, p. 180-183. In B. Klinge and C. Owman (Eds.), BioScience, 
Lund University Press, Lund.

18.Rigler, R., J. Widengren and U. Mets. 1992. Interaction and kinetics of single molecules as observed by fluorescence correlation spectroscopy, p. 13-24. In O.S. Wolfbeis (Ed.), Fluorescence Spectroscopy: New Methods and Applications. Springer-Verlag, Berlin.

19.Schwille, P., F. Oehkenschläger and N.G. Walter. 1996. Quantitative hybridization kinetics of DNA probes to RNA in solution followed by diffusional fluorescence correlation analysis. Biochemistry 35:10182-10193.

20.Thompson, N. 1991. Fluorescence correlation spectroscopy, p. 337-378. In J.R. Lakowicz (Ed.), Topics in Fluorescence Spectroscopy. 1. Plenum Press, New York and London.

21.Walter, N.G., P. Schwille and M. Eigen. 1996. Fluorescence correlation analysis of probe diffusion simplifies quantitative pathogen detection by PCR. Proc. Natl. Acad. Sci. USA 93:12805-12810.

22.Widengren, J., Ü. Mets and R. Rigler. 1995. Fluorescence correlation spectroscopy of triplet states in solution: a theoretical and experimental study. J. Phys. Chem. 99:1336813379 .

Received 29 December 1997; accepted 27 March 1998.

Address correspondence to:

Dr. Masataka Kinjo

Laboratory of Biophysics

Research Institute for Electronic Science

Hokkaido University

060, Sapporo, Japan

Internet:injo@imd.es.hokudai.ac.jp 